Pacific Journal of Mathematics

A NOTE ON PRIMARY DECOMPOSITIONS OF A 


\title{
A NOTE ON PRIMARY DECOMPOSITIONS OF A PSEUDOVALUATION
}

\author{
C. P. L. RHODES
}

Some connections are established between a primary decomposition of a pseudovaluation $v$ on a commutative ring and a primary decomposition of the zero ideal of the associated graded ring of $v$. The primary decomposition of a certain pseudovaluation $v_{q}$ on a one-dimensional local ring $Q$ is described in terms of the extensions of $v_{\mathfrak{q}}$ to monoidal transforms of $Q$.

1. Primary decompositions and the associated graded ring. Let $R$ be a commutative ring with an identity. We consider a pseudovaluation $v$ on $R$. By this we mean that $v$ is a mapping from $R$ to $\boldsymbol{P}$, the set of all real numbers together with $\infty$, such that

$$
v(0)=\infty, \quad v(1)=0,
$$

and, for $x, y \in R$,

and

$$
v(x y) \geqq v(x)+v(y),
$$

$$
v(x-y) \geqq \min \{v(x), v(y)\} \text {. }
$$

For each $a \in \boldsymbol{P}$, write $v_{a}=\{x \in R \mid v(x) \geqq a\}$ and $v_{\bar{a}}=\{x \in R \mid v(x)>a\}$. The associated graded ring of $v$, introduced by Szpiro in [11], is $G=\bigoplus_{a \in \boldsymbol{R}} v_{a} / v_{\bar{a}}$. We shall use - to denote the natural mapping from $R$ into $G$.

Let $u$ be a pseudovaluation such that $u \geqq v$ (this means that $u(x) \geqq v(x)$ for all $x)$. We denote by $T(u)$ the set of all $x$, not in $u_{\infty}$, such that $u\left(x^{n}\right)=n u(x)$ for all positive integers $n$, and by $S(u)$ the set of all $x$, not in $u_{\infty}$, such that $u(x y)=u(x)+u(y)$ for all $y \in R$. As in [10], we call $u$ primary if $T(u)=S(u)$. We denote by $F(u, v)$ the set of all $x$ such that either $u(x)>v(x)$ or $u(x)=\infty$, and we put $T(u, v)=T(u) \backslash F(u, v)$.

Let $I(u, v)$ be the ideal generated in $G$ by $\overline{F(u, v)}$.

Lemma 1. $\overline{F(u, v)}$ is the set of all homogeneous elements of $I(u, v)$, and $\overline{T(u, v)}$ is the set of all homogeneous elements of $G \backslash \operatorname{rad} I(u, v)$. If the pseudovaluation $u$ is primary then the ideal $I(u, v)$ is primary.

Proof. Let $r \in R$ and $s \in F(u, v)$. Either $\bar{r} \bar{s}=\overline{0}$ or $\bar{r} \bar{s}=\overline{r s}$. In the latter case either $v(s)=\infty$ or

$$
v(r s)=v(r)+v(s)<u(r)+u(s) \leqq u(r s) .
$$


Thus, in each case, $\bar{r} \bar{s} \in \overline{F(u, v)}$. If we suppose, also, that $r \in F(u, v)$ and that $\bar{r}$ and $\bar{s}$ have the same degree, then either $\bar{r}-\bar{s}=\overline{0}$ or $\bar{r}-\bar{s}=\overline{r-s}$. In the latter case either $v(r)=\infty$ or

$$
v(r-s)=v(r)=v(s)<\min \{u(r), u(s)\} \leqq u(r-s) .
$$

Hence, in each case, $\bar{r}-\bar{s} \in \overline{F(u, v)}$. It is now clear that $\overline{F(u, v)}$ is the set of homogeneous elements of $I(u, v)$.

Let $r \in T(u, v)$ and let $n$ be a positive integer. Then it is easy to see that $u\left(r^{n}\right)=v\left(r^{n}\right)=n v(r) \neq \infty$; i.e., $r^{n} \notin F(u, v)$. Therefore, $\bar{r}^{n}=\overline{r^{n}} \notin I(u, v)$. Now suppose that $r \notin T(u, v)$. If $r \notin T(v)$ then there exists $m$ such that $\bar{r}^{m}=\overline{0}$. Suppose that $r \in T(v)$. Then, by 4.1 of [10], there exists $n$ such that $r^{n} \in F(u, v)$. Hence $\bar{r}^{n}=\overline{r^{n}} \in I(u, v)$.

Finally let $u$ be primary, and suppose that $r, s$ are elements of $R$ such that $r \in T(u, v), \bar{s} \neq \overline{0}$, and $\bar{r} \bar{s} \in I(u, v)$. Either $\bar{r} \bar{s}=\overline{0}$ and so $v(r)+v(s)<v(r s) \leqq u(r s)=u(r)+u(s)=v(r)+u(s)$, or $\bar{r} \bar{s}=\overline{r s}$ and so $v(r)+v(s)=v(r s)<u(r s)=u(r)+u(s)=v(r)+u(s)$. In each case $v(s)<u(s)$ and, hence, $\bar{s} \in I(u, v)$. Therefore, $I(u, v)$ is primary.

REMARK. The set $S(u) \backslash F(u, v)$ is contained in the set $S_{0}(u, v)$ of all $x \notin F(u, v)$ such that $u(x y)=u(x)+u(y)$ for all $y \notin F(u, v)$. These sets and their images in $G$ are multiplicatively closed, and $\overline{S_{0}(u, v)}$ is the set of all homogeneous elements of $G$ which are relatively prime to $I(u, v)$.

If $W$ is a collection of pseudovaluations the lower envelope $w_{0}=$ $\Lambda W$ is defined by $w_{0}(x)=\inf \{w(x) \mid w \in W\}$. From Lemma 1 we deduce

THEOREM 1. If $u_{1} \wedge u_{2} \wedge \cdots \wedge u_{n}$ is a primary decomposition of $v$ then $I\left(u_{1}, v\right) \cap I\left(u_{2}, v\right) \cap \cdots \cap I\left(u_{n}, v\right)$ is a primary decomposition of $0_{G}$.

COROLLARY. Let $u_{1} \wedge u_{2} \wedge \cdots \wedge u_{n}$ be an irredundant primary decomposition of $v$, and suppose that $G$ is Noetherian. Then, for each $i$, there exists $r_{i} \in R$ such that $T\left(u_{i}, v\right)$ is the set of $x$, not in $v_{\infty}$, for which $v\left(x r_{i}\right)=v(x)+v\left(r_{i}\right)$.

Proof. The decomposition $0_{G}=I\left(u_{1}, v\right) \cap \cdots \cap I\left(u_{n}, v\right)$ is clearly irredundant. It follows that the homogeneous elements of $G$ not in $\overline{T\left(u_{i}, v\right)}$ generate a prime ideal which belongs to $0_{G}$ and which, therefore, takes the form $0_{G}:\left(G \bar{r}_{i}\right)$ for some homogeneous element $\bar{r}_{i}$ in $G$. 
REMARK. For each positive $b \in \boldsymbol{P}$, denote by $F(u, v, b)$ the set of all $r \in R$ such that either $u(r)=\infty$ or $u(r)-v(r) \geqq b$. The proof of Lemma 1 shows that $\overline{F(u, v, b)}$ is the set of homogeneous elements of the ideal $I(u, v, b)$ which it generates in $G$, and that $\overline{T(u, v)}$ is the set of homogeneous elements of $G \backslash \operatorname{rad} I(u, v, b)$. It is easy to verify that, for a (possibly infinite) collection of pseudovaluations $v_{i} \geqq v, v=\bigwedge_{i} v_{i}$ if and only if, for every $b>0,0_{G}=\bigcap_{i} I\left(v_{i}, v, b\right)$.

For all $b>0$ and $c>0$,

$$
I(u, v, b) I(u, v, c) \subseteq I(u, v, b+c) .
$$

Hence each $u \geqq v$ naturally induces a (nonnegative) pseudovaluation $u^{\prime}$ on $G$. Thus $v=\Lambda_{i} v_{i}$ if and only if $\Lambda_{i} v_{i}^{\prime}$ is the trivial pseudovaluation on $G$.

When $v$ is homogeneous the following result may be regarded as a special case of [11, Théorème 1]. Recall that $v$ is said to be discrete if $v\left(R \backslash v_{\infty}\right)$ generates a discrete subgroup of $\boldsymbol{R}$.

THEOREM 2. Suppose that $v$ is a discrete pseudovaluation. If $0_{G}$ has a finite primary decomposition without embedded components then $v$ has a primary decomposition.

Proof. Suppose that $H_{1} \cap H_{2} \cap \cdots \cap H_{l}$ is the primary decomposition of $0_{G}$. For each $i$, write $\operatorname{rad} H_{i}=P_{i}$ and denote by $S_{i}$ the set of elements $r \in R$ such that $\bar{r} \notin P_{i}$; then $v(a b)=v(a)+v(b)$ for all $a$ and $b$ in $S_{i}$, and $S_{i}$ is multiplicatively closed. Mappings $v_{i}$ are defined, for all $x \in R$, by

$$
v_{i}(x)=\sup \left\{v(x a)-v(\alpha) \mid a \in S_{i}\right\} .
$$

Observe that if $a, b \in S_{i}$ then

$$
v_{i}(x) \geqq v(x a b)-v(a b) \geqq v(x a)-v(a) \geqq v(x) .
$$

By 3.1 and 3.2 of [6], $v_{i}$ is a pseudovaluation.

Let $x \in R \backslash v_{\infty}$. Then there exists $i$ such that $\bar{x} \in H_{i}$. If $c \in S_{i}$ then $\bar{x} \bar{c} \neq \overline{0}$, and so $v(x c)-v(c)=v(x)$. Thus $v_{i}(x)=v(x)$, and so $\Lambda_{i} v_{i}=v$.

We shall now show that $v_{1}$, being a typical $v_{i}$, is primary. Let $x \in S\left(v_{1}\right)$ and suppose that $v_{1}(x) \neq \infty$. Then there exists $y \in R$ such that $v_{1}(x y)>v_{1}(x)+v_{1}(y)$. Therefore, we may choose $a \in S_{1}$ such that

$$
v_{1}(x)=v(x a)-v(\alpha),
$$

and

$$
v_{1}(x y) \geqq v(x y a)-v(a)>v_{1}(x)+v_{1}(y) \cdot
$$


Now write $\bigcap_{i>1} P_{i}=K$ and choose $c \in R$ such that $\bar{c} \in K \backslash P_{1}$. Then $\bar{a} \bar{c} \notin P_{1}$, and so $\overline{a c}=\bar{a} \bar{c} \in K \backslash P_{1}$. We may therefore assume (by replacing $a$ by $a c$ ) that $\bar{a} \in K \backslash P_{1}$. This implies that $\overline{a^{2}}=\bar{a} \bar{a} \in K \backslash P_{1}$. Since $v_{1}(x)=v\left(x a^{2}\right)-v\left(a^{2}\right)=v(x a)-v(a)$, it follows that

$$
v\left(x a^{2}\right)=v(x a)+v(a) \text {. }
$$

Therefore, $\overline{x a^{2}}=\overline{x a} \bar{a} \in K$, and so (replacing $a$ by $a^{2}$ ) we may also assume that $\overline{x a} \in K$. If $\overline{x a} \notin P_{1}$ then

$$
v(x y a)-v(a)=v(y x a)-v(x a)+v(x a)-v(\alpha) \leqq v_{1}(y)+v_{1}(x),
$$

which is false. Therefore, $\overline{x a} \in \bigcap_{i \geqq 1} P_{i}$, and so, for some $n,(\overline{x a})^{n}=0_{G}$. Since $v_{1}(x) \neq \infty$, we have $v(x a) \neq \infty$ and so $v\left((x a)^{n}\right)>n v(x a)$. Therefore, $v_{1}\left(x^{n}\right) \geqq v\left(x^{n} a^{n}\right)-v\left(a^{n}\right)>n v(x a)-v\left(a^{n}\right)=n v_{1}(x)$. Thus $x \notin T\left(v_{1}\right)$. Therefore, $S\left(v_{1}\right)=T\left(v_{1}\right)$; i.e., $v_{1}$ is primary.

2. Extensions of pseudovaluations. In this section we introduce some terminology for use in $\S 3$, and we prove a result pertinent to [2].

We suppose the definition of a pseudovaluation $u$ to be modified as follows:

(i) $u \geqq 0$.

(ii) It is not required that $u(1)=0$ (this facilitates the statement of Lemma 2; moreover, the rings in this section need not contain an identity).

We consider a homomorphism $f$ from a commutative ring $R$ to a commutative ring $S$. If $I$ is an ideal of $S\left(\right.$ resp. $R$ ) then $I^{c}$ (resp. $I^{e}$ ) will denote $f^{-1} I$ (resp. the ideal generated by $f(I)$ in $S$ ). Suppose that $v$ is a pseudovaluation on $R$. Define $v^{e}$ to be the mapping from $S$ to $P$ such that, for all $x \in S$,

$$
v^{e}(x)=\sup \left\{a \in \boldsymbol{P} \mid x \in\left(v_{a}\right)^{e}\right\} .
$$

LeMma 2. The mapping $v^{e}$ is a pseudovaluation on $S$.

Proof. It is clear that $v^{e}(0)=\infty$.

Let $x, y \in S$, and suppose that $x \in\left(v_{a}\right)^{e}$ and $y \in\left(v_{b}\right)^{e}$ where $a, b \in \boldsymbol{P}$. Then $x y \in\left(v_{a}\right)^{e}\left(v_{b}\right)^{e} \cong\left(v_{a} v_{b}\right)^{e} \cong\left(v_{a+b}\right)^{e}$. Thus

$$
v^{e}(x y) \geqq a+b \text {. }
$$

It follows that $v^{e}(x y) \geqq v^{e}(x)+v^{e}(y)$.

Similarly, assuming that $a \geqq b, x-y \in\left(v_{a}\right)^{e}+\left(v_{b}\right)^{e}=\left(v_{a}+v_{b}\right)^{e}=$ $\left(v_{b}\right)^{e}$. Thus $v^{e}(x-y) \geqq b$. It follows that 


$$
v^{e}(x-y) \geqq \min \left\{v^{e}(x), v^{e}(y)\right\}
$$

Let $w$ be a pseudovaluation on $S$. We shall denote $w f$ by $w^{c}$. It is easy to verify that $w^{c}$ is a pseudovaluation on $R$ which is primary if $w$ is primary.

Lemma 3. (i) The pseudovaluation $v$ on $R$ satisfies $v \leqq v^{e c}$.

(ii) The pseudovaluation $w$ on $S$ satisfies $w \geqq w^{c e}$.

Proof. (i) Let $x$ be an element of $R$ such that $v(x)=a$. Then $f(x) \in\left(v_{a}\right)^{e}$ and so $a \leqq v^{e}(f(x))=v^{e c}(x)$.

(ii) Let $y$ be an element of $S$ such that $y \in\left(\left(w^{c}\right)_{a}\right)^{e}$. Since $\left(w^{c}\right)_{a} \subseteq\left(w_{a}\right)^{c}, y \in\left(w_{a}\right)^{c e} \subseteq w_{a}$ and so $w(y) \geqq a$. It follows that $w(y) \geqq$ $w^{c e}(y)$.

THEOREM 3. $v=v^{e c}$ if and only if $v_{a}=\left(v_{a}\right)^{e c}$ for each $a \in \boldsymbol{R}$.

Proof. If $v=v^{e c}$ then, for each $a \in P$,

$$
\left(v_{a}\right)^{e c} \leqq\left\{x \in R \mid v^{e}(f(x)) \geqq a\right\}=\left(v^{e c}\right)_{a}=v_{a},
$$

and so $\left(v_{a}\right)^{e c}=v_{a}$. Conversely, suppose that $v_{a}=\left(v_{a}\right)^{e c}$ for each $a \in \boldsymbol{R}$. Let $x \in R$ and let $f(x) \in\left(v_{a}\right)^{e}$ where $a<\infty$. Then $x \in\left(v_{a}\right)^{e c}=$ $v_{a}$, that is $v(x) \geqq a$. It follows that $v \geqq v^{e c}$, and hence that $v=v^{e c}$.

We refer to [2, p. 296, Definition 2] for the definition of a best filtration. If $v$ has a best filtration $\left\{A_{i}\right\}_{i=0}^{\infty}$ then, by $[2, \mathrm{p} .297$, Lemma 1], the set of all distinct $A_{i}$ 's is the same as the set of all distinct $v_{a}$ 's where $a<\infty$. Thus, taking $f$ to be an inclusion map, our theorem includes, in the case of nonnegative pseudovaluations, Theorem 2, p. 299, and Theorem 4, p. 301, of [2].

3. An example in a one-dimensional ring. Let $Q, \mathfrak{m}$ be a one-dimensional local ring and let $\mathfrak{q}$ be an m-primary ideal of $Q$. We shall consider the pseudovaluation $v=v_{\mathrm{q}}$ determined by the powers of $q$ according to the rule

$$
v_{\mathfrak{q}}(x)=\sup \left\{n \mid x \in \mathfrak{q}^{n}\right\} .
$$

By considering the associated graded ring $G$ of $v$ and proceeding as in Theorem 2, we could show that $v$ decomposes into primary pseudovaluations corresponding to the isolated primary components of $0_{G}$ together with an "irrelevant" component. Apart from the irrelevant component this decomposition is unique (by [10]). We shall now show how the theory of monoidal transformations developed by Northcott and Kirby provides an alternative description of this 
decomposition.

Let $A$ denote the intersection of the primary components of $0_{Q}$ of rank nought, and write $Q / A=Q^{\prime}$ and $\mathfrak{q} Q^{\prime}=\mathfrak{q}^{\prime}$. Then not every element of $m Q^{\prime}$ is a zero divisor. Let $\Re$ be the $q^{\prime}$-resolute of $Q^{\prime}$, for the definition of which see p. 136 of [4]; let $Q_{1}, \cdots, Q_{r}$ be the monoidal transforms of $Q^{\prime}$ with respect to $\mathfrak{q}^{\prime}$, i.e., the rings of quotients of $\Re$ with respect to the maximal ideals $\mathfrak{p}_{1}, \cdots, \mathfrak{p}_{r}$ of $\Re$; and, for $i=1, \cdots, r$, let $f_{i}$ be the composition of the natural homomorphisms $Q \rightarrow Q^{\prime} \rightarrow Q_{i}$. Using the symbols $e_{i}$ and $c_{i}$ to relate to $f_{i}$ in the same way that $e$ and $c$ were related to $f$ in $\S 2$, we observe that $v^{e_{i}}$ is the pseudovaluation on $Q_{i}$ determined the powers of the ideal $\mathfrak{q}^{e_{i}}$. However, by [4, Theorems 1 and 8, and Lemma 3] $\mathfrak{q}^{e_{i}}$ is a principal ideal of $Q_{i}$. Therefore, by an example in $\S 3$ of [10], $v^{e_{i}}$ is primary, and so $v^{e_{i} c_{i}}$ is primary.

Now, denoting by $\mathfrak{q}_{i}$ the restriction to $\Re$ of $\mathfrak{q}^{e_{i}}, \operatorname{rad} \mathfrak{q}_{i}=\mathfrak{p}_{i}$ and $\mathfrak{q}_{1} \cap \mathfrak{q}_{2} \cap \cdots \cap \mathfrak{q}_{r}$ is the primary decomposition of $\mathfrak{R q}$ (by the corollary on p. 142 of [4] and since $\Re m \subseteq \operatorname{rad} \Re q)$. Therefore, for all $n$,

$$
\Re \mathfrak{q}^{n}=\mathfrak{q}_{1}^{n} \cap \mathfrak{q}_{2}^{n} \cap \cdots \cap \mathfrak{q}_{r}^{n} .
$$

By an argument on p. 88 of [8], $\Re q^{n}=\mathfrak{q}^{\prime n}$ for all sufficiently large $n$. Therefore, for $n \geqq h$ say,

$$
\mathfrak{q}^{n}+A=\left(v^{e_{1} c_{1}} \wedge \cdots \wedge v^{e_{r} r^{r}}\right)_{n} .
$$

However, we may choose $h$ such that $A \cap q^{h}=0_{Q}$ and, hence, for $n \geqq h, q^{h} \cap\left(q^{n}+A\right)=q^{n}$. Therefore, using $e_{0}$ and $c_{0}$ to relate to the natural map $f_{0}$ from $Q$ to $Q / \mathfrak{q}^{h}$, we have, for all $n$,

$$
\mathfrak{q}^{n}=\left(v^{e_{0} c_{0}}\right)_{n} \cap\left(v^{e_{1} c_{1}} \wedge \cdots \wedge v^{e} r^{c} r\right)_{n} .
$$

Finally we show that $v^{e_{0}},=w$ say, is primary. If $x \in f_{0}(\mathfrak{m})$ then, for some $k, w\left(x^{k}\right)=\infty$ and so $x \notin T(w)$. On the other hand, if $x$ is a unit of $Q / \mathfrak{q}^{h}$ then $w(x)=0$ and, for any $y$,

$$
w(x y)=w(y)=w(y)+w(x) ;
$$

i.e., $x \in S(w)$. Thus $T(w)=S(w)$.

It is now clear that

THEOREM 4. In the notation developed above

$$
v^{e_{0} c_{0}} \wedge v^{e_{1} c_{1}} \wedge \cdots \wedge v^{e_{r} c_{r}}
$$

is a primary decomposition of $v$.

It is easy to extend this theorem and obtain a primary decomposition of the pseudovaluation $v_{I}$ determined by an ideal $I$ of rank 1 
in a 1-dimensional Noetherian ring $R$. Let $M_{1}, \cdots, M_{m}$ be the associated prime ideals (necessarily maximal) of $I$, and, for $j=1, \cdots, m$, let $g_{j}$ be the natural homomorphism from $R$ to the ring $R_{j}$ of quotients of $R$ with respect to $M_{j}$. For each positive integer $n$,

$$
I^{n}=\bigcap_{j}\left(I^{n}\right)^{e_{j} c_{j}}
$$

where $e_{j}, c_{j}$ relate to $g_{j}$, and so

$$
v_{I}=\bigwedge_{j} v_{I}^{e^{j} c_{j}}
$$

which yields a primary decomposition of $v_{I}$ on application of Theorem 4 to each $v_{I}^{i}$.

We conclude by describing a result, in the same vein as the foregoing, which is implicit, as a special case, in [9]. Suppose that our ring $R$ is a domain; let $\bar{v}_{I}$ denote the least homogeneous pseudovaluation $\geqq v_{1}$; and let $\bar{R}_{1}, \cdots, \bar{R}_{h}$ be the rings of quotients with respect to the maximal ideals of the integral closure of $R$ which contain $I$. Then $\bar{v}_{I}$ decomposes into valuations

$$
\bar{v}_{I}=\Lambda_{i}\left(\overline{v_{I}^{e}}\right)^{c_{i}}
$$

where, for each $i, e_{i}, c_{i}$ refer to the natural mapping $R \rightarrow \bar{R}_{i}$.

\section{REFERENCES}

1. G. M. Bergman, A weak nullstellensatz for valuations, Proc. Amer. Math. Soc., 28 (1971), 32-38.

2. J. A. Huckaba, Extensions of pseudo-valuations, Pacific J. Math., 29 (1969), 295-302.

3. - Some results on pseudo-valuations, Duke Math. J., 37 (1970), 1-9.

4. D. Kirby, Monoidal transformations in a one-dimensional local ring, Mathematika, 9 (1962), 135-144.

5. K. Kiyek, Ein Struktursatz für Pseudobewertungen, Math. Z., 118 (1970), 139-156.

6. - Homogene Pseudobewertungen, Arch. Math., 22 (1971), 602-611.

7. D. G. Northcott, On the notion of a first neighbourhood ring with an application to the $A F+B \Phi$ theorem, Proc. Camb. Phil. Soc., 53 (1957), 43-56.

8. - The reduction number of a one-dimensional local ring, Mathematika, 6 (1959), 87-90.

9. D. Rees, Valuations associated with ideals (II), J. London Math. Soc., 31 (1956), 221-228.

10. C. P. L. Rhodes, The partial uniqueness of a primary decomposition of a pseudovaluation, J. London Math. Soc., (2), 6 (1973), 231-240.

11. L. Szpiro, Fonctions d'ordre et valuations, Bull. Soc. Math. France, 94 (1966), 301311.

Received May 15, 1972.

University College, Cardiff, Wales, U. K. 



\section{PACIFIC JOURNAL OF MATHEMATICS}

\section{EDITORS}

D. Gilbarg AND J. MILGRAM

Stanford University

Stanford, California 94305

\section{R. A. Beaumont}

University of Washington

Seattle, Washington 98105

\section{J. DUGUNDJI*}

Department of Mathematics University of Southern California Los Angeles, California 90007

RICHARD ARENS

University of California Los Angeles, California 90024

\section{ASSOCIATE EDITORS}
E. F. BECKENBACH
B. H. NEUMANN
F. WOLF
K. YoshidA

\section{SUPPORTING INSTITUTIONS}

\author{
UNIVERSITY OF BRITISH COLUMBIA \\ CALIFORNIA INSTITUTE OF TECHNOLOGY \\ UNIVERSITY OF CALIFORNIA \\ MONTANA STATE UNIVERSITY \\ UNIVERSITY OF NEVADA \\ NEW MEXICO STATE UNIVERSITY \\ OREGON STATE UNIVERSITY \\ UNIVERSITY OF OREGON \\ OSAKA UNIVERSITY
}

\author{
UNIVERSITY OF SOUTHERN CALIFORNIA \\ STANFORD UNIVERSITY \\ UNIVERSITY OF TOKYO \\ UNIVERSITY OF UTAH \\ WASHINGTON STATE UNIVERSITY \\ UNIVERSITY OF WASHINGTON \\ AMERICAN MATHEMATICAL SOCIETY \\ NAVAL WEAPONS CENTER
}

The Supporting Institutions listed above contribute to the cost of publication of this Journal, but they are not owners or publishers and have no responsibility for its content or policies.

Mathematical papers intended for publication in the Pacific Journal of Mathematics should be in typed form or offset-reproduced, (not dittoed), double spaced with large margins. Underline Greek letters in red, German in green, and script in blue. The first paragraph or two must be capable of being used separately as a synopsis of the entire paper. Items of the bibliography should not be cited there unless absolutely necessary, in which case they must be identified by author and Journal, rather than by item number. Manuscripts, in duplicate if possible, may be sent to any one of the four editors. Please classify according to the scheme of Math. Rev. Index to Vol. 39. All other communications to the editors should be addressed to the managing editor, Richard Arens, University of California, Los Angeles, California, 90024.

50 reprints are provided free for each article; additional copies may be obtained at cost in multiples of 50 .

The Pacific Journal of Mathematics is issued monthly as of January 1966. Regular subscription rate: $\$ 48.00$ a year (6 Vols., 12 issues). Special rate: $\$ 24.00$ a year to individual members of supporting institutions.

Subscriptions, orders for back numbers, and changes of address should be sent to Pacific Journal of Mathematics, 103 Highland Boulevard, Berkeley, California, 94708.

PUBLISHED BY PACIFIC JOURNAL OF MATHEMATICS, A NON-PROFIT CORPORATION

Printed at Kokusai Bunken Insatsusha (International Academic Printing Co., Ltd.), 270, 3-chome Totsuka-cho, Shinjuku-ku, Tokyo 160, Japan.

* C. DePrima will replace J. Dugundji until August 1974.

Copyright (C) 1973 by

Pacific Journal of Mathematics

All Rights Reserved 


\section{Pacific Journal of Mathematics}

\section{Vol. 47, No. $2 \quad$ February, 1973}

David Parham Bellamy, Composants of Hausdorff indecomposable continua; a mapping approach ........................ 303

Colin Bennett, A Hausdorff-Young theorem for rearrangement-invariant spaces ...........................................

Roger Daniel Bleier and Paul F. Conrad, The lattice of closed ideals and $a^{*}$-extensions of an abelian l-group ...

Ronald Elroy Bruck, Jr., Nonexpansive projections on subsets of Banach

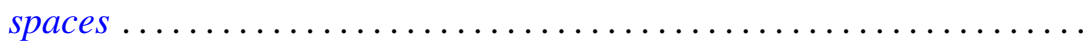

Robert C. Busby, Centralizers of twisted group algebras ............. 357

M. J. Canfell, Dimension theory in zero-set spaces ................ 393

John Dauns, One sided prime ideals ........................ 401

Charles F. Dunkl, Structure hypergroups for measure algebras . . . . . . . . . 413

Ronald Francis Gariepy, Geometric properties of Sobolev mappings ...... 427

Ralph Allen Gellar and Lavon Barry Page, A new look at some familiar spaces of intertwining operators ...........................

Dennis Michael Girard, The behavior of the norm of an automorphism of the

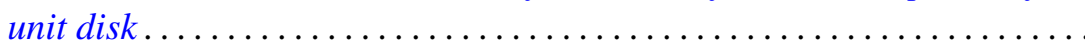

George Rudolph Gordh, Jr., Terminal subcontinua of hereditarily

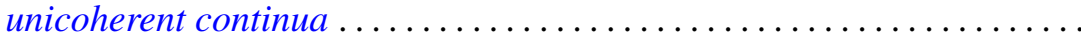

Joe Alston Guthrie, Mapping spaces and cs-networks. .

Neil Hindman, The product of $F$-spaces with $P$-spaces . 473

M. A. Labbé and John Wolfe, Isomorphic classes of the spaces $C_{\sigma}(S)$

Ernest A. Michael, On k-spaces, $k_{R}$-spaces and $k(X) \ldots$

Donald Steven Passman, Primitive group rings .

C. P. L. Rhodes, A note on primary decompositions of a pseudovaluation ...

Muril Lynn Robertson, A class of generalized functional differential equations

Ruth Silverman, Decomposition of plane convex sets. $I$.

Ernest Lester Stitzinger, On saturated formations of solvable Lie algebras................................

B. Andreas Troesch, Sloshing frequencies in a half-space by Kelvin inversion ...

L. E. Ward, Fixed point sets .

Michael John Westwater, Hilbert transforms, and a problem in scattering

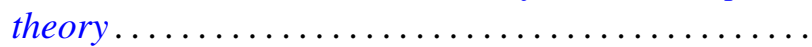

Misha Zafran, On the spectra of multipliers... 